\title{
Therapy Monitoring of Oncologic Disease in the Abdomen (Including PET/CT)
}

\author{
Irene A. Burger and Regina G. H. Beets-Tan
}

\section{Learning Objectives}

- To understand the opportunities and limitations of the different modalities for therapy monitoring based on anatomical and physiological imaging

- To know the pearls and pitfalls in therapy monitoring of gastrointestinal stromal tumors, neuroendocrine tumors, and metastasized prostate cancer

- To learn about the evidence, pearls, and pitfalls of imaging for the local assessment of response to neoadjuvant (chemo)radiotherapy in locally advanced rectal tumors

\subsection{Introduction}

The importance and the volume of scans for therapy monitoring are steadily increasing for oncologic diseases. Numerous reasons account for that. Most important is the occurrence of an increasing number of new and more specific therapies requiring early outcome measures preceding first results on survival benefits. Furthermore, the specific therapeutic approaches concomitant with longer therapy windows lead to an increase in mixed response results, requiring reliable evaluation by imaging approaches.

Therefore, an increasing need emerged, and focus has been put on how to best evaluate such early onset of therapeutic efficacy. In this chapter we will provide a brief overview on the most commonly used imaging modalities, their

I. A. Burger $(\square)$

Department of Nuclear Medicine, UniversitätsSpital Zürich,

Zürich, Switzerland

e-mail: Irene.burger@usz.ch

R. G. H. Beets-Tan

Department of Radiology, The Netherlands Cancer Institute, Amsterdam, The Netherlands

e-mail: r.beetstan@nki.nl benefits and their pitfalls focusing on the evaluation of therapeutic efficacy with CT, MRI, and molecular imaging (PET/CT) of several abdominal tumors such as GIST, neuroendocrine tumors, and metastatic prostate cancer.

Furthermore, the increase of neoadjuvant therapy for high-risk tumors significantly improved outcome in various tumor entities. Here imaging is essential not only for overall response assessment but also to determine resectability. Therefore we will address the importance of endorectal ultrasonography (ERUS) and MRI in locally advanced rectal tumors treated with neoadjuvant (chemo)radiotherapy.

\subsection{Assessment of Local Response to Neoadjuvant Treatment in Rectal Cancer}

The local recurrence rate after rectal cancer surgery has significantly decreased from $40 \%$ three decades ago to $3 \%$ with current multimodality imaging and therapy. Three major steps have been taken forward: the introduction of the total mesorectal excision (TME) surgery, preoperative radiotherapy with or without chemotherapy, and imaging. The risk factors associated with local recurrence are the $\mathrm{T}$ stage, $\mathrm{N}$ stage, distance of the tumor to the mesorectal fascia, extramural vascular invasion, perineural invasion, lymph vessel invasion, and histological grade. Rectal cancer patients are now treated according to their risk for local recurrence with a differentiated treatment-immediate TME surgery for the low-risk tumors and preoperative neoadjuvant chemoradiotherapy for the very high risks. The decisions on preoperative treatment are based on risk assessment not only by digital rectal examination and endoscopy but also by imaging. Endorectal ultrasonography (ERUS) and MRI are considered as the two best locoregional imaging methods to assess the risk factors at primary staging and to evaluate local response to neoadjuvant treatment, while CT is restricted to distant staging. Here we'll focus on local response evaluation with MR and ERUS. 


\subsubsection{Selection of Patients for Neoadjuvant Therapy}

The importance of the involvement of the mesorectal fascia as a prognostic factor and as a parameter of surgical quality has been recognized and confirmed in the last 30 years [1]. The ideal plane of resection in a total mesorectal excision is just outside the mesorectal fascia, and a positive circumferential resection margin can be the result of inadequate TME surgery. Preoperative assessment of involvement of the mesorectal fascia is crucial. An involved mesorectal fascia can be reliably determined on MRI and is defined as a closest distance of $\leq 1 \mathrm{~mm}$ between the tumor and the mesorectal fascia. These tumors should be treated with a preoperative (chemo)radiation regimen providing an opportunity for downsizing. Regardless of the choice for neoadjuvant treatment strategies, it is however important for the surgeon to know the exact anatomical relation of the tumor to the mesorectal fascia and the surrounding structures in order to obtain a complete resection, and MRI can provide this information. A number of studies in the past decades demonstrated that MRI could reliably identify the mesorectal fascia and predict its involvement [2, 3]. In a European multicenter study, the depth of extramural tumor invasion was compared between preoperative MRI and histology in 311 patients undergoing primary surgery; accuracy to within $0.5 \mathrm{~mm}$ was achieved in $95 \%$ of cases [4]. It is now widely adopted that MRI is the most accurate method for the assessment of an involved or threatened mesorectal fascia (Fig. 4.1).
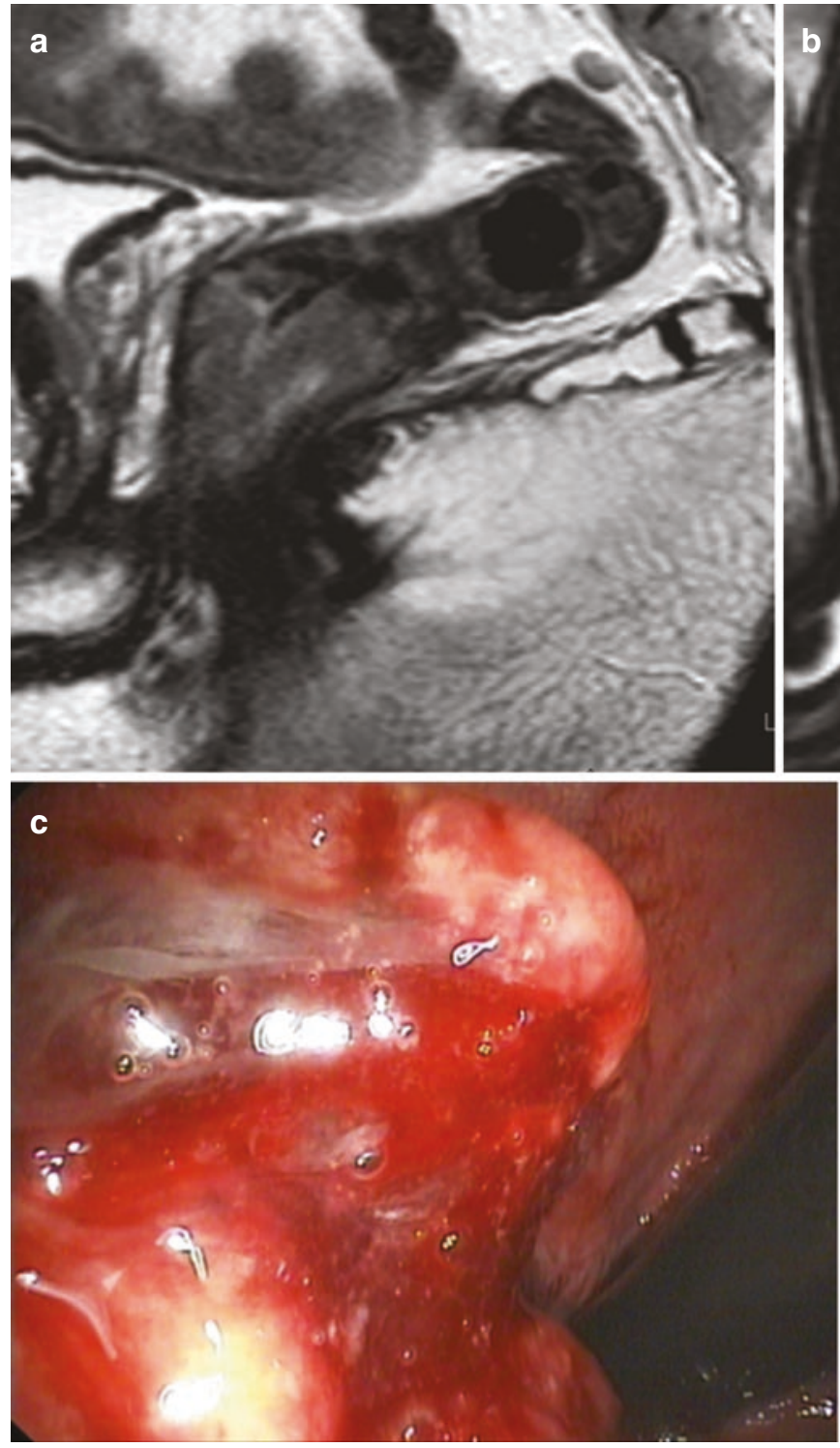

Fig. 4.1 cT2N2 low rectal tumor before neoadjuvant chemoradiotherapy. Endoscopy shows a sessile semicircular mass anteriorly located just above the anal canal (a-d). T2-weighted MRI shows a semicircular tumor low anterior which is limited to the bowel wall. There is a suspi-

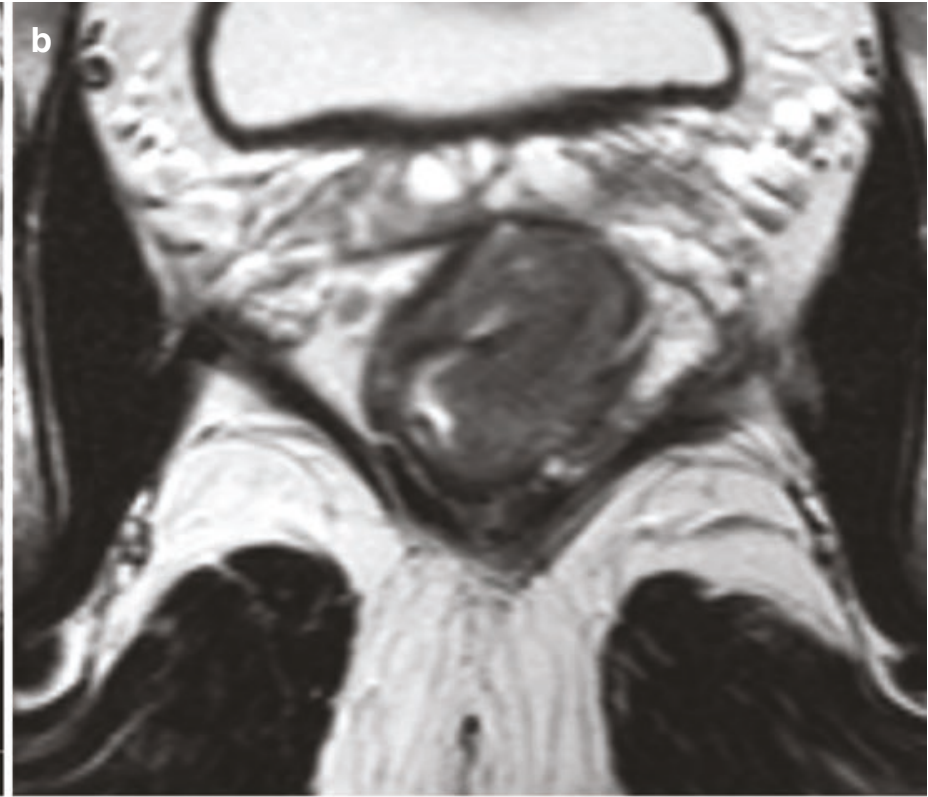

d

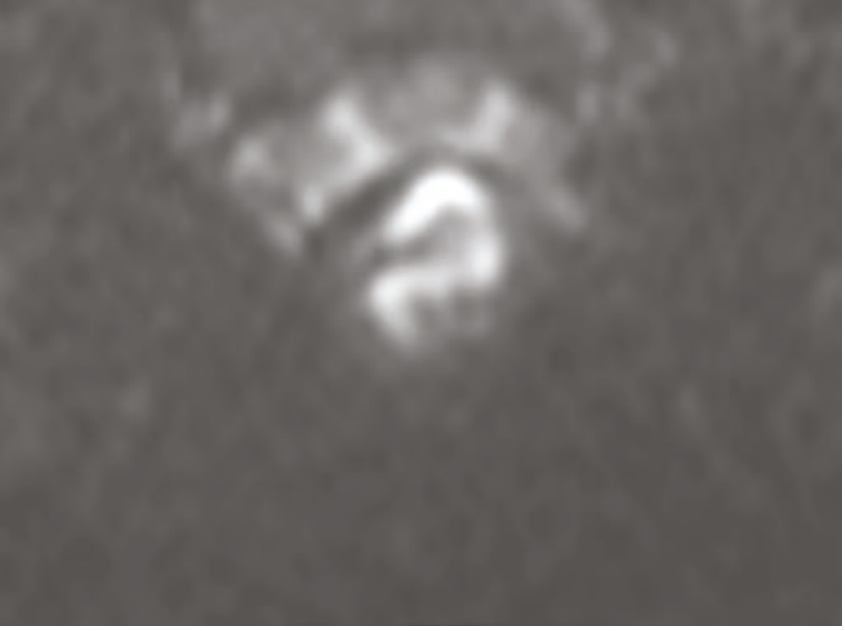

cious large node in the mesorectum, visualized on the sagittal T2-weighted MR image. DWI (b1000) image confirms a rectal cancer with a restricted diffusion 


\subsubsection{Assessment of Local Tumor Response and Resectability}

Assessment of local tumor response is generally performed by digital rectal examination, endoscopy, and MRI 6-8 weeks after completion of neoadjuvant chemoradiotherapy [5]. MRI can indicate tumor volume shrinkage. Reported negative predictive values (NPVs) as high as $90 \%$ for the detection of an involved mesorectal fascia suggest that MRI can accurately identify clearance of an initially suspicious invaded mesorectal resection margin. The high NPV is at the expense of a PPV as low as 50\%. As a result of the irradiation, the initial tumor mass often becomes fibrotic, and it may be very difficult to distinguish on T2-weighted MRI between fibrosis with and without minimal residual tumor. This holds true also for fibrotic masses adjacent to the mesorectal fascia, the reason why in such a setting one should be cautious to overcall persistent involvement of a mesorectal fascia after chemoradiotherapy (Fig. 4.2) [6, 7].

Restaging MRI can therefore be valuable as a road map for surgeons and to decide whether tumor regression visualized on MRI justifies alteration of the initial resection plan. The obvious prerequisite for the value of a restaging MRI is that both the surgeon and the patient must be willing to change the initial plan.

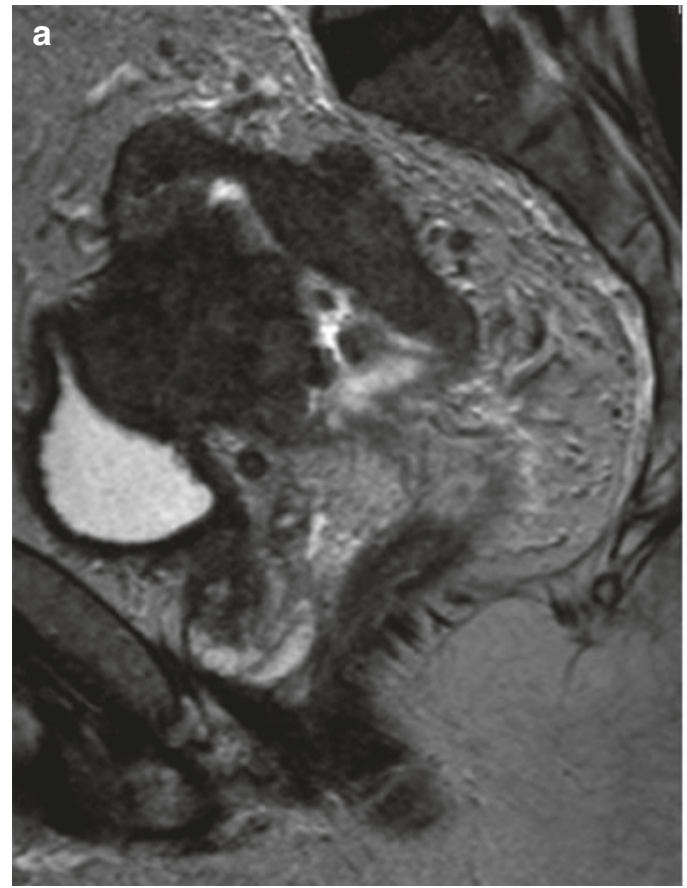

Fig. 4.2 T2-weighted MRI of a locally advanced rectal tumor before (a) and after (b) neoadjuvant chemoradiotherapy. The tumor located in the mid-high rectum anteriorly invades the peritoneal reflection and shows a mass effect. It can often be difficult to know whether the tumor is invading in or only pushing the bladder wall and dome. After chemoradiotherapy the tumor has significantly shrunk and for a large propor-

\subsubsection{Assessment of Complete Response After Chemoradiotherapy}

Rectal cancer patients with clinical complete or near complete response after chemoradiotherapy have shown to have a good oncological outcome with a nonoperative management with survival figures as good as when these patients would have undergone a resection. This treatment shows an improved functional outcome without compromising oncological outcome. Habr-Gama et al. and the group of Beets et al. were pioneers of these socalled watch and wait policy $[8,9]$. Today more and more patients with clinical complete response are considered a watch and wait policy.

A watch and wait treatment for the clinical complete or near complete responders requires accurate assessment of response to chemoradiotherapy and accurate monitoring of response. Although this is generally done by digital rectal examination, imaging has shown its benefit. The two important questions are whether there is still a significant volume of residual tumor within the fibrotic tumor bed and whether there are still involved nodes after CRT. When it comes to primary $\mathrm{T}$ staging, MRI is the preferred staging method for large advanced tumors, while ERUS remains the best method to image the superficial tumors limited to the submucosa (T1) [10].

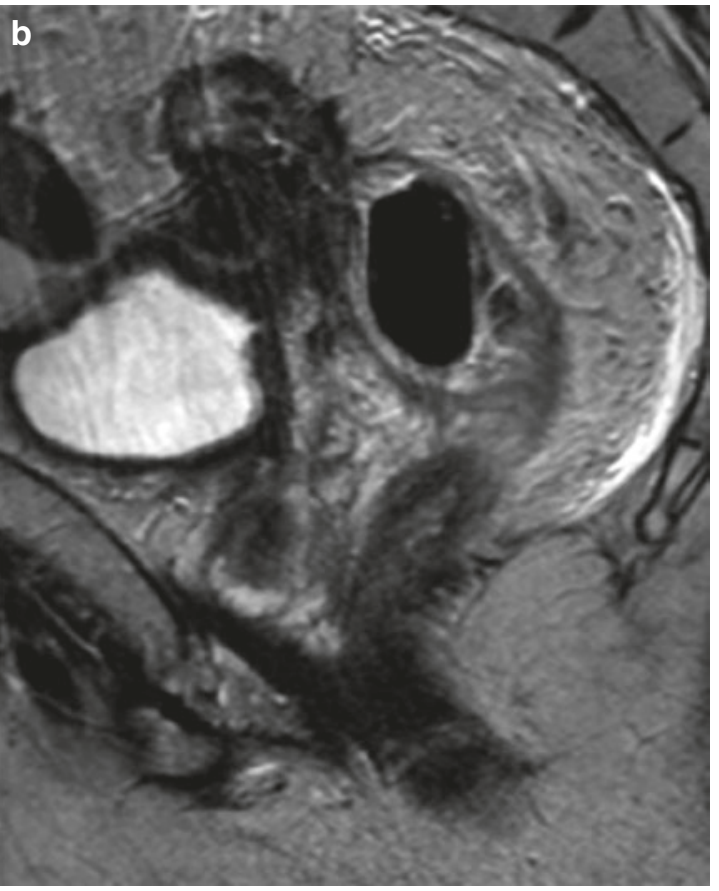

tion becomes fibrotic. Anteriorly it has retracted from the bladder dome but not from the peritoneal reflection, and there is a fibrotically thickened peritoneal reflection. The surgeon should be notified of this finding so that he can perform a wider excision including the peritoneal reflection 
Nevertheless, for assessment of the ypT stage of rectal tumors after neoadjuvant treatment, MRI does not outperform ERUS. A meta-analysis of over 1500 patients reported a disappointingly low overall performance with a pooled overall sensitivity of 50\% and specificity of $91 \%$ [11]. Subgroup analyses showed a pooled sensitivity of only $19 \%$ and specificity of $94 \%$ for the MRI assessment of pathological complete response (ypT0). Although the performance of MRI for assessment of small tumor remnants limited to the bowel wall (ypT0-2) is higher, the sensitivity of $55 \%$ remains insufficient for clinical decision-making. The findings above suggest that both ERUS and MRI are limited in the detection of small tumor remnants within fibrotic tissue after neoadjuvant chemoradiotherapy of rectal cancer. Some authors have shown that the combination of visual assessment of morphological features on post-chemoradiotherapy MRI with objective assessment of tumor shrinkage by magnetic resonance volumetric measurements can yield accuracies as high as $87 \%[12,13]$. Curvo-Semedo et al. and Ha et al. found AUCs (areas under the ROC curve) of 0.70-0.84 for the assessment of pathological complete response with magnetic resonance volumetric measurements by manual delineation of tumors [14]. This is very time-consuming and thus not a standard practice. Many studies have reported the benefit of diffusion-weighted MRI (DW-MRI) for the detection of residual disease within the fibrotic scar. A meta-analysis has shown that DW-MRI can improve the diagnostic performance of restaging MRI for the assessment of a pathological complete response with an increase in sensitivity from $19 \%$ to $84 \%$ [11]. A multicenter retrospective study in 120 patients with rectal cancer reported a high NPV of $90 \%$ for DW-MRI for the assessment of a pathological complete response, indicating that the visual analysis of DW-MRI is especially valuable for the detection of residual tumor and for ruling out a complete response [15]. Visual analysis of DW-MRI (at b800 or b1000) is now recommended in international clinical guidelines as a valuable adjunct to digital rectal exam and endoscopy. Patients assessed as (near) complete response with these tools can be safely considered for a watch and wait treatment [5]. Figure 4.3 shows the typical DW-MR and endoscopic image of a clinical complete response.

Overall, there is little evidence concerning restaging with ERUS after neoadjuvant treatment. In a study by Mezzi et al. and Napoleon et al., ERUS after neoadjuvant treatment was only accurate in $47 \%$ and $46 \%$ of patients, respectively. Fibrotic, necrotic, and inflammatory changes after preoperative radiotherapy can lead to misinterpretation of ERUS images [16, 17]. Radovanovic et al. found a good accuracy rate for restaging rectal cancer after neoadjuvant chemoradiation (75\%) [18]. However, ERUS was insufficient in discriminating a complete pathological response, in fact the only key factor of importance in clinical decision-making, as described by the groups of Habr-Gama and Beets [8, 9]. The largest study, by Pastor et al., reported an accuracy of only
$47 \%$ for the ERUS assessment of complete response. $53 \%$ of tumors identified as yT0 by ERUS were understaged [19].

\subsubsection{Assessment of Response for Nodal Disease}

Node-positive disease is one of the most important risk factors for both local and distant recurrence and is generally considered an indication for neoadjuvant therapy. Identifying mesorectal nodal disease with any imaging technique remains difficult because size criteria used on its own result in only a moderate accuracy. The majority of mesorectalinvolved nodes are smaller than $5 \mathrm{~mm}$ resulting in inaccuracies when size is used as a cutoff for nodal disease. Meta-analyses showed no significant differences between the three modalities, EUS, CT, or MRI, which were all associated with sensitivities for primary staging around 60-70\% [20]. Because the majority of mesorectal nodes disappear or shrink after chemoradiotherapy, restaging nodes after CRT can be more accurate with known improved sensitivities around 70-75\%. Nevertheless, assessment of nodal involvement in rectal cancer in general remains a challenging task for any imaging method, and at the moment of writing, this problem has not been solved.

\subsection{Assessment of Response for Systemic Disease}

The World Health Organization (WHO) recognized the need for standardized criteria across clinical trials very early, publishing the initial WHO handbook for reporting results of cancer treatment in 1979 [21]. Since then a number of guidelines and updates have been published.

\subsubsection{Response Based on Morphology for Chemotherapy}

The most commonly applied response criteria for systemic disease are Response Evaluation Criteria in Solid Tumors (RECIST) that have been updated to RECIST 1.1 [22]. Based on the changes of target and nontarget lesions, patients are categorized into four groups: complete response (CR), partial response $(\mathrm{PR})$, stable disease (SD), and progressive disease (PD). To simplify readouts only five target lesions (TL), maximum of two in the same organ, shall be assessed by the maximum diameter. Lymph nodes are measured by the short axis and need to be larger than $1.5 \mathrm{~cm}$ to be considered as target lesions. Sclerotic bone lesions are considered nontarget lesions (NTL), as well as cystic lesions, if they do not have large solid components. The sum of all diameters from 



Fig.4.3 DW-MRI and endoscopy of a clinical complete response after neoadjuvant chemoradiotherapy. Endoscopy shows complete regression of the in Fig. 4.1 visualized tumor with only a white scar tissue with telangiectasia at the irradiated tumor bed (a-d). T2-weighted MRI shows a minimal fibrotic wall anteriorly without any distortion of the wall anatomy. DWI (b1000) image shows no restricted diffusion in the tumor bed. On the sagittal T2-weighted MR image, the initially suspicious node has decreased in size and shows no suspicious features of malignancy. The patient was successfully treated and closely monitored within a watch and wait regimen. Ten years in follow-up still alive without recurrent disease

\subsubsection{Response Based on Morphology for Immunotherapy}

Classical chemotherapies directly kill tumor cells leading to shrinkage of tumor volume based on the efficacy of the therapy. Immunotherapies activate the immune system of the patient with different response patterns depending on the mechanism of drug action [23]. Summarized morphologic response based on immunotherapy will often need more time compared to conventional therapies, and response patterns can be grouped into four categories: 
(1) immediate response, (2) durable stable disease, (3) response after initial increase (flare), and (4) response with new lesions.

For accurate interpretation of the response pattern, understanding of the drug mechanism is crucial. Therapeutics leading to an increasing migration of $\mathrm{T}$ cells into the tumor (CTLA-4 blocking, e.g., ipilimumab) will be more likely to have an initial increase in tumor size [24]. iRECIST is based on RECIST 1.1 to select TL and NTL; if however PD is seen on first follow-up, this is interpreted as iUPD (unconfirmed progressive disease); only if tumor increase of more than $30 \%$ is confirmed on the second follow-up (4-8 weeks later), a confirmed progression iCPD will be noted. If the tumor remains stable, iUPD will remain the interpretation, and only if criteria for partial or complete response are met, iPR or iCR will be given [23].

Besides accurate interpretation of early pseudoprogression, the recognition of immune-related response (irR) is crucial as well. Inflammatory changes can result in activation of sarcoidosis with enlarged lymph nodes or adrenalitis with enlarged suprarenal glands. This is not only challenging for morphologic imaging but also for PET/ CT since irR can show intensive ${ }^{18} \mathrm{~F}$-fluorodeoxyglucose (FDG) uptake [25]. Immunotherapies are already a clinically established option for patients with melanoma or lung cancer. However, more indications also for abdominal diseases will follow soon, such as prostate cancer (sipuleucel-T), kidney cancer (nivolumab), or bladder cancer (pembrolizumab).

\subsubsection{Response Based on FDG PET}

Numerous publications showed a good correlation between the decrease in FDG accumulation in tumor lesions and therapy response [26]. Therefore, PET response evaluation was postulated, including EORTC PET response recommendations (1999) and the PET response criteria in solid tumors (PERCIST) pioneered by Wahl et al. in 2009 [27]. Both methods follow the model of RECIST with four adapted response categories: complete metabolic response (CMR), partial metabolic response (PMR), stable metabolic disease (SMD), and progressive metabolic disease (PMD). Numerous studies compared the original EORTC PET criteria with PERCIST in the past 8 years showing similar results. However, the use of PERCIST seems preferable for clinical trials due to a better standardization [28]. Both methods recommend the use of standardized uptake values (SUV) normalized to the lean body mass (SUL). However, if possible PERCIST favors the use of a SUL peak (average value in the hottest $1 \mathrm{~cm}^{3}$ sphere within the tumor), instead of $\mathrm{SUL}_{\max }$ (only one maximum voxel) to reduce the intrinsic variability.

\begin{tabular}{|c|c|}
\hline Response & PERCIST-Based on $\mathrm{SUL}_{\text {peak }}\left(\mathrm{SUL}_{\max }\right)$ \\
\hline PMD & $\begin{array}{l}\text { SUL increase by at least } 30 \% \text { and at least } 0.8 \text { SUL } \\
\text { units of the target lesion or increase in target lesion size } \\
\text { by } 30 \% \text { or development of at least one new lesion or } \\
\text { unequivocal progression of nontarget lesions }\end{array}$ \\
\hline SMD & Increase or decrease of SUL by less than $30 \%$ \\
\hline PMR & $\begin{array}{l}\text { Decrease of SUL by } \geq 30 \% \text { and at least } 0.8 \text { SUL unit } \\
\text { difference and no new FDG-avid lesions and no increase } \\
\text { in size }>30 \% \text { of the target lesion and no increase in SUL } \\
\text { or size of nontarget lesion }\end{array}$ \\
\hline CMR & $\begin{array}{l}\text { FDG uptake indistinguishable from surrounding } \\
\text { background and SUL less than the liver }\end{array}$ \\
\hline
\end{tabular}

It is important to note that all these recommendations are focusing on FDG PET for the evaluation of metabolic response of solid tumors. Tumor dedifferentiation does not correlate with receptor expression in the same way like FDG. Therefore, PERCIST per se is not necessarily applicable for the increasing use of receptor imaging in oncologic diseases, e.g., somatostatin receptor II (SSTR II) for neuroendocrine tumors or prostate-specific membrane antigen (PSMA) for prostate cancer.

\subsection{Monitoring GIST Molecular Targeted Systemic Therapy}

\subsubsection{Monitoring GIST with CT/MRI}

With the introduction of tyrosine kinase inhibitors imatinib mesylate $\left(\right.$ Glivec $\left.^{\circledR}\right)$ in 2002 for gastrointestinal stromal tumors (GIST), a new area of molecular-targeted systemic therapy began, followed by a variety of targeted agents for various indications [29, 30]. Those new therapeutic approaches result in new patterns regarding response assessment, with the classical reduction in tumor size assessed by RECIST criteria often being detected weeks to months later [31]. Classical response patterns that can be observed in liver metastasis include appearance of new lesions, increase in size with decrease in attenuation, increase in attenuation (internal hemorrhage), and temporal changes. These patterns can be explained by the primary effect on tumor vascularity leading to hypoenhancement and therefore pseudoprogression on contrast-enhanced CT scans in the classical portovenous phase, with combination of intratumoral edema and internal hemorrhages [32]. To integrate these observations into clinical response assessment, Choi et al. suggested that either a decrease in size by $10 \%$ (largest diameter) or a decrease in attenuation by $15 \%$ on portovenous contrastenhanced CT should be rated as response [33]. More recent approaches for optimal response assessment for GIST included the use of dual-energy CT, with calculation of iodine-related attenuation as a superior predictor for response compared to simple density used for the Choi criteria [34]. 
MRI can be used in undetermined cases to detect intratumoral hemorrhage and to assess vascularity of the lesions. Furthermore Tang et al. showed that an increase in ADC values after 1 week of therapy is associated with good treatment response [35]. Both methods dual-energy CT and MRI have not been clinically validated to date.

\subsubsection{Monitoring GIST with PET}

In the first safety and efficacy report on imatinib (2001) including 40 patients, the use of FDG PET was documented for a subgroup of 17 patients, where the decrease in FDG uptake correlated well with the outcome and preceded CT findings according to RECIST criteria [29] (Fig. 4.4). This led to numerous studies integrating FDG for response assessment with variable cutoff values to indicate response. Therefore, the ESMO Clinical Practice Guidelines for GIST suggest the use of FDG PET/CT for early detection of tumor response under targeted therapy [36]. Choi et al. used modified EORTC criteria of a decrease in $\mathrm{SUV}_{\max }$ of more than $70 \%$ or an absolute value of $\mathrm{SUV}_{\max }<2.5$ as a cutoff [33]. Holdsworth et al. investigated optimal cutoffs for FDG PET based on 63 patients using ROC analysis and suggested a decrease of $\mathrm{SUV}_{\max }$ of more than $40 \%$ or an absolute value of
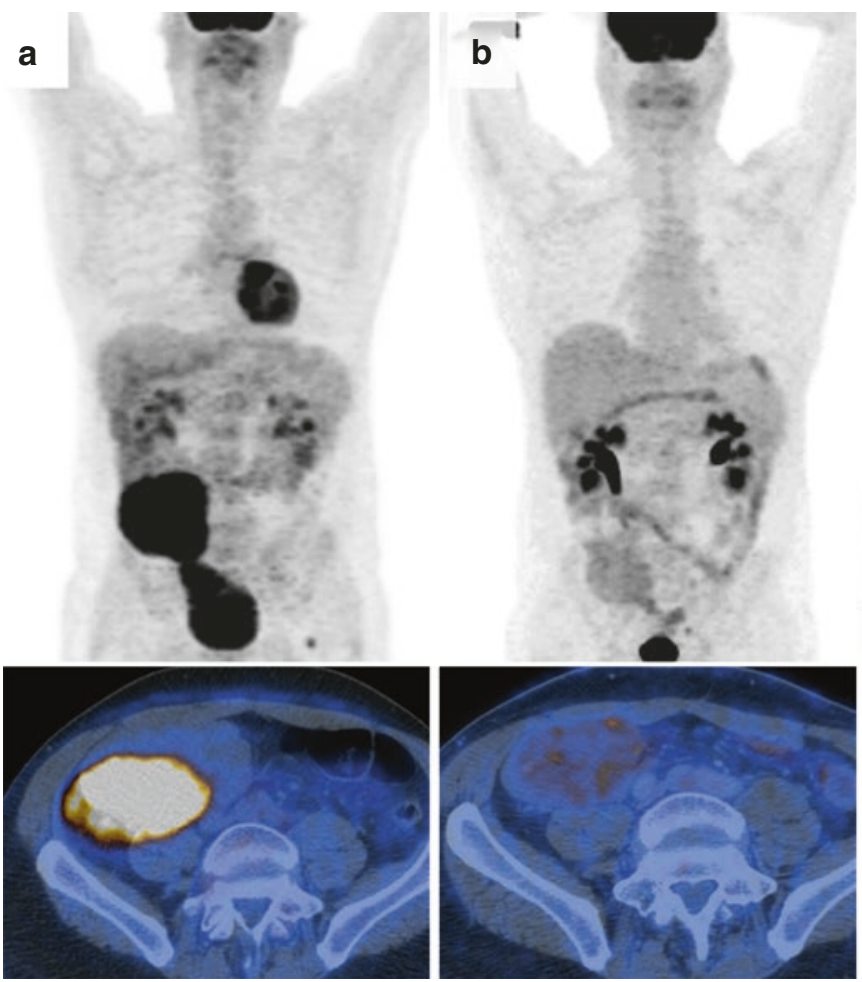

Fig. 4.4 MIP FDG PET and fused axial FDG PET/CT images of two patients with highly avid GIST tumors. The first patient presented with metastatic small bowel GIST (a) and showed subtotal response after 4 weeks of therapy with imatinib (b), with only minimal decrease in
$\mathrm{SUV}_{\max }<3.4$ outperformed EORTC criteria in their cohort [37]. Nevertheless, the most commonly used cutoff for new drug trials is still EORTC criteria with a decrease of $>25 \%$ of $\mathrm{SUV}_{\max }$ indicating partial response [38]. Recently published data from the Dutch GIST registry on the impact of FDG PET displayed a changed patient management in $27 \%$ of GIST patients, due to a lack of metabolic response. Furthermore, the registry data showed that especially patients without KIT exon 11 mutations had limited response to tyrosine kinase inhibitors, with a change in management based on FDG PET in 52\% of these patients [39].

\subsection{Monitoring Liver Disease After SIRT}

Selective internal radiotherapy (SIRT) using yttrium-90 $\left.{ }^{90} \mathrm{Y}\right)$ resins or glass microspheres is an increasingly used palliative therapy option for patients with non-resectable primary liver tumors or metastatic hepatic disease. Clinical evaluation of patients prior to SIRT includes contrastenhanced CT of the chest and the abdomen to rule out extensive extrahepatic disease, liver MRI to assess tumor burden, and selective angiography to evaluate vascular anatomy and hepatic shunt with technetium- $99 \mathrm{~m}$ labeled aggregated macroalbumin $\left({ }^{99 \mathrm{~m}} \mathrm{Tc}-\mathrm{MAA}\right)$ [40].
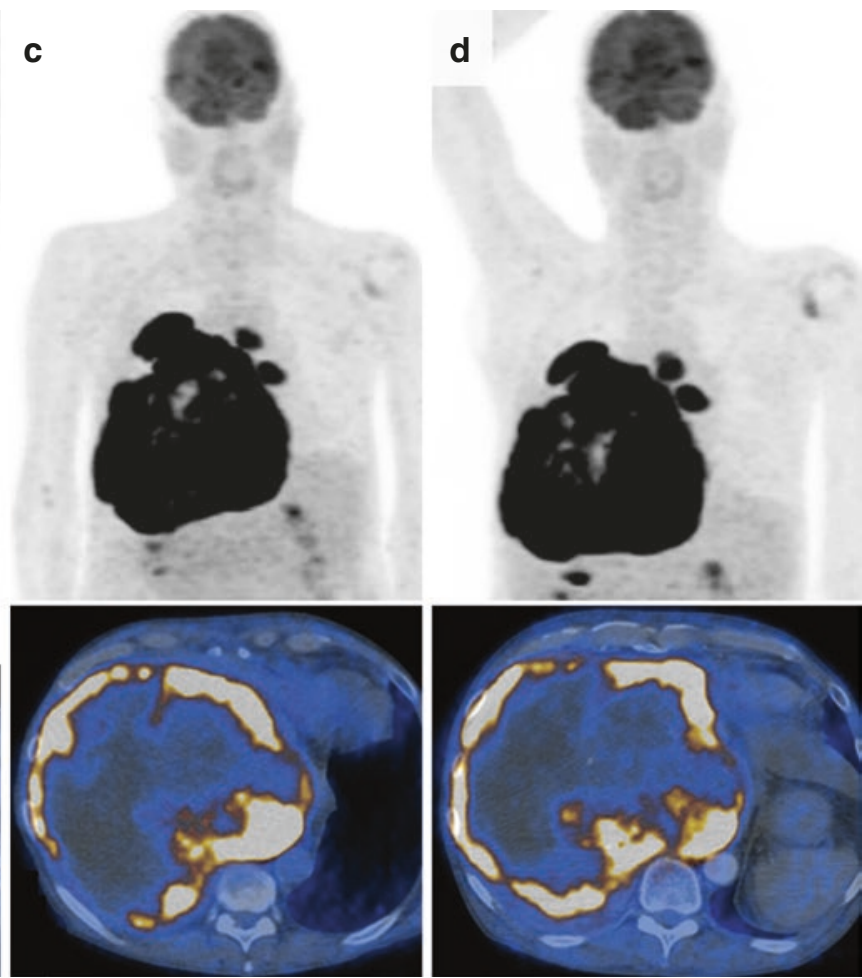

size. For the second patient with extensive pleural GIST manifestation (c), follow-up PET after 4 weeks showed no response to neoadjuvant imatinib (d), followed by immediate surgical resection 


\subsubsection{Monitoring SIRT with CT/MRI}

Early response assessment after SIRT using anatomical imaging can be limited due to delayed reduction in tumor size and initial pseudoprogression due to edema and sharper demarcation of tumor boundaries on conventional imaging (Fig. 4.5). Early investigations with serial contrast enhanced CT (ceCT) images showed a maximum decrease in tumor size at 3-21 months (median 12 months), concluding that blood tumor markers (e.g., CEA for colorectal metastasis) were superior in therapy response assessment compared to contrast-enhanced CT [41].

The calculation of arterial perfusion (AP) using dynamic contrast-enhanced CT prior to SIRT was the best predictor for good treatment response and overall survival in patients [42]. Furthermore, follow-up perfusion CT showed a significant decrease of AP in hepatic metastasis 4 weeks after SIRT in patients with long-term response, compared to nonresponders [43]. Dual-energy CT is a new technology, allowing the creation of iodine maps as promising tools to evaluate and quantify tumor viability, utilizing iodine maps measuring the amount of iodine per lesion. Although this approach requires validation and standardization, it showed promising first results for hepatic radiofrequency ablation [44] and could also be a promising tool for SIRT therapy response assessment.

Functional MRI was suggested to be used to assess response to SIRT, as well. A post-therapeutic increase in $\mathrm{ADC}_{\text {min }}$ of more than $22 \%, 4$ weeks after SIRT, was significantly associated with a superior overall survival (18 vs. 5 months, $p<0.001$ ), while tumor size did not show any significant decrease after 4 weeks [45].

\subsubsection{Monitoring SIRT with PET}

Early on FDG PET was used to assess the reduction of hepatic metastatic load after SIRT [46]. Studies comparing response assessment between FDG PET/CT and ceCT gen-
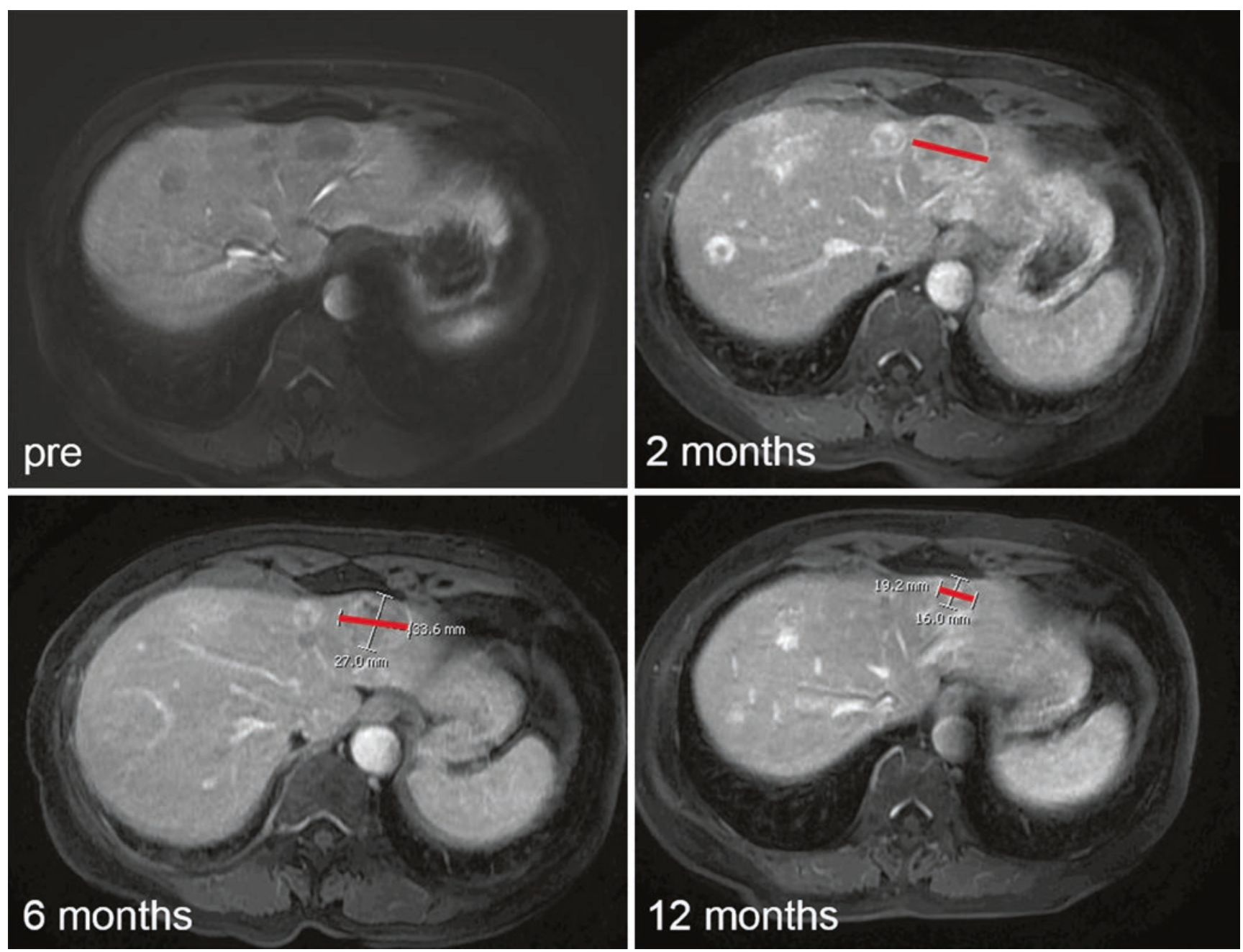

Fig.4.5 Axial post-contrast MRI before and 2, 6, and 12 months after SIRT therapy, showing the long-standing pseudoprogression over 6 months, with improved delineation of metastases on the first scan after SIRT and final partial morphologic response after 12 months 
erally show a higher predictive value for FDG PET compared to ceCT [47], as published, e.g., in a review article in 2014, where PET/CT revealed superiority over ceCT using RECIST for early response assessment [48]. Other groups investigated the PET-based volume metrics such as the metabolic tumor volume (MTV) or the total lesion glycolysis (TLG) rate and came to the conclusion that those parameters correlate better with outcome compared to plain tumor size or $\mathrm{SUV}_{\max }$ [49] measured in the PET scan. For accurate response assessment with FDG PET, potential pitfalls have to be considered. The two most prominent limitations are false-negative results caused by partial volume effects in small lesions $(<1 \mathrm{~cm})$ or diabetes and false-positive results caused by abscess formation or inflammatory changes [50]. A combination of FDG PET/CT with ceCT can reduce these limita-tions.

Despite the fact that earlier prediction of response to SIRT with FDG PET is possible, there is no established clinical role for FDG PET in assessing tumor response after SIRT. This might be attributable to a lack of additional treatment options. Therefore, early knowledge of a limited response to this palliative therapy will change treatment only in very few cases. Nevertheless, it is crucial to know the potential limitations and possibilities of the various imaging modalities to prevent false interpretation of early morphologic changes for accurate judgment of therapeutic response.

\subsection{Monitoring Neuroendocrine Tumors}

Well-differentiated neuroendocrine tumors (NET) are slowgrowing lesions and therefore usually assessed with contrastenhanced CT scans for staging and detection of progression. New therapeutic approaches such as the combination of everolimus with octreotide long-acting repeatable are systematically assessed based on progression-free survival according to RECIST and chromogranin A blood values [51]. Also the recently published NETTER-1 trial investigated the efficacy of peptide-based systemic radiotherapy (SRT) with ${ }^{177} \mathrm{Lu}$-Dotatate based on conventional imaging. Tumor progression was defined as a primary endpoint documented with either CT or MRI. The secondary endpoint was overall survival. An increase of progression-free survival from $10.8 \%$ in the control group to $65.2 \%$ in the ${ }^{177} \mathrm{Lu}$-Dotatate group was observed after 20 months. On interim analysis of survival, the estimated risk of death was $60 \%$ lower in the ${ }^{177} \mathrm{Lu}$-Dotatate group than in the control group [52].

Similar to SIRT and targeted therapies, the morphologic response lags behind physiologic changes and pseudoprogression due to edema, and improved tumor delineation of liver lesions can be observed. This is crucial, since progression under therapy is a potential reason to stop current treat- ment according to the European Neuroendocrine Tumor Society (ENETS) consensus guidelines. Therefore, a harmonization and combination of anatomical and molecular imaging as well as biomarkers was suggested for monitoring SRT [53]. No standardized procedures have been established yet on how to interpret molecular imaging (e.g., ${ }^{68} \mathrm{Ga}$-DOTATATE PET/CT) results after SRT. Only one publication summarizing results of 33 patients undergoing early therapy response assessment with ${ }^{68} \mathrm{Ga}$-DOTATATE after 1 cycle of SRT was published so far. They came to the conclusion that a decrease of the tumor to spleen ratio $\left(\mathrm{SUV}_{\mathrm{T} / \mathrm{S}}\right)$ correlated well with progression-free survival $(p=0.002)$, while a decrease in $\mathrm{SUV}_{\text {max }}$ did not reach significance [54] (Fig. 4.6).

\subsection{Monitoring Metastasized Prostate Cancer (I)}

\subsubsection{Conventional Monitoring of Metastasized Prostate Cancer with CT and Bone Scans}

Imaging response assessment for prostate cancer is notoriously difficult on anatomical imaging and therefore plays only a secondary role for treatment evaluation in new drug trials that commonly focus on survival and PSA values instead [55, 56]. For the trials investigating efficacy of abiraterone and alpharadin $\left({ }^{223} \mathrm{Ra}\right)$, progressive disease was defined as an increase in PSA values or a morphologic progression according to RECIST (lymph nodes $(>2 \mathrm{~cm}$ ) or visceral metastasis selected as target lesions; progression defined as an increase of $>20 \%$ of target lesions or two or more new lesions on bone scans not consistent with tumor flare). Bone scans were included since around $68 \%$ of prostate cancer patients will develop bone metastasis, and most of these lesions are unmeasurable disease according to RECIST [57]. It is well established that bone scans have a higher sensitivity for bone metastasis compared to CT. However, healing bone metastases will initially react with increasing mineralization and therefore also with an increase in activity on ${ }^{99 \mathrm{~m}} \mathrm{Tc}$-bone scans or ${ }^{18} \mathrm{~F}$-flouride PET/CT the so-called tumor flare. This typically lasts for 3 months after therapy but can be seen as late as 6 months after treatment [57]. Therefore, ${ }^{99 \mathrm{~m}} \mathrm{Tc}$-bone scans and ${ }^{18} \mathrm{~F}-$ flouride PET/CT alone are both limited for response assessment in prostate cancer patients. To still be able to include bone lesions for response assessment, bone-specific response criteria were suggested by MD Anderson incorporating CT and MRI information indicative for response (e.g., sclerotic fill-in or rim of lytic lesions) together with a decrease of tracer uptake on bone scans to improved evaluation of bone lesions [58] (Fig. 4.7). 
a

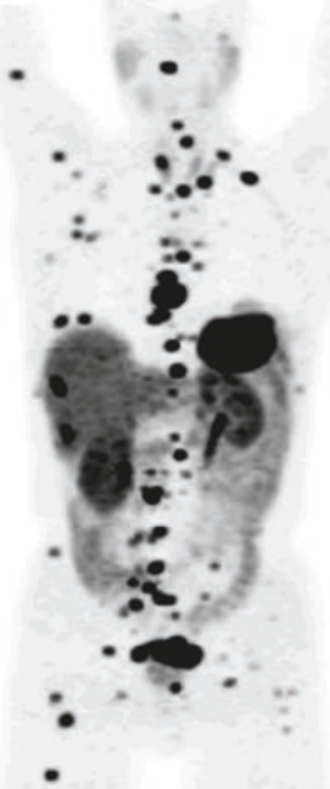

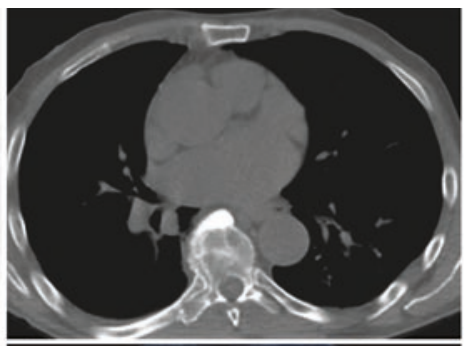
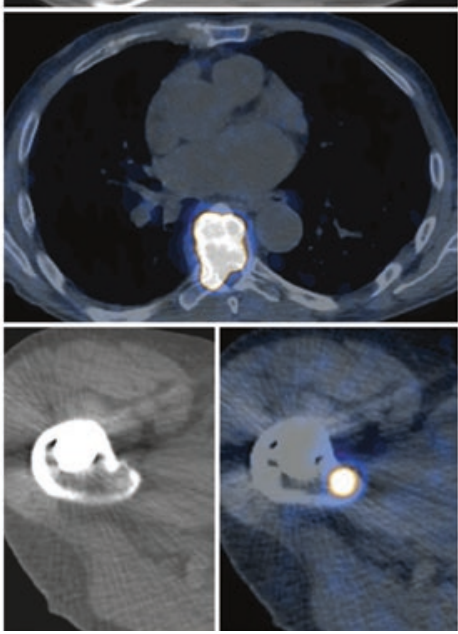

b
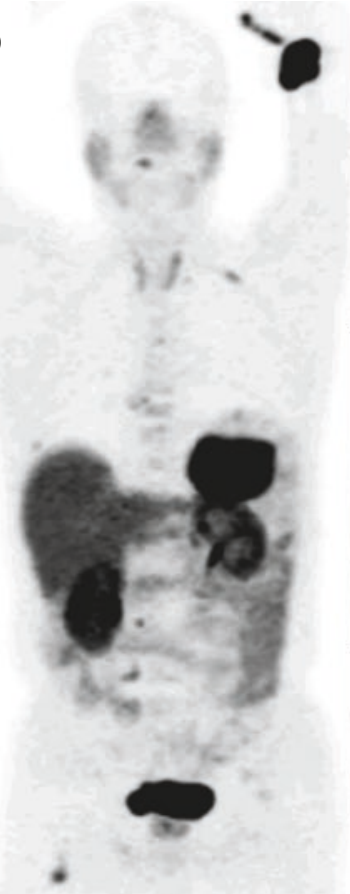
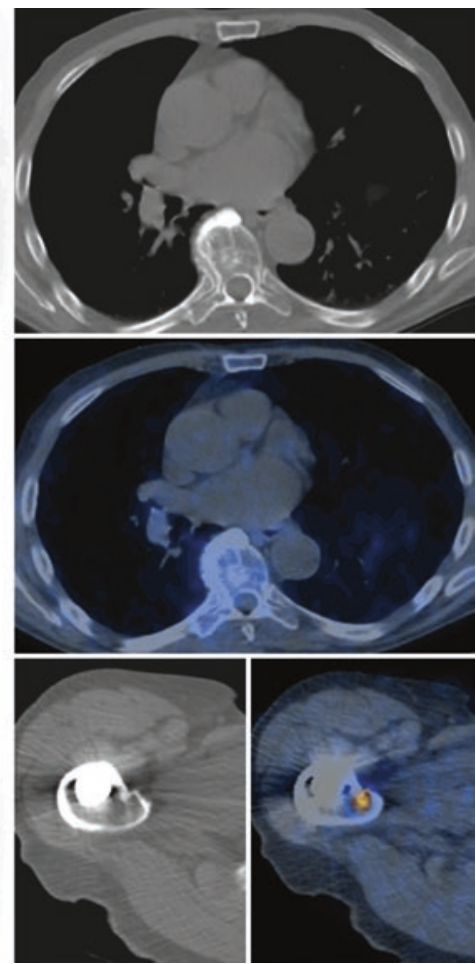

Fig. 4.6 ${ }^{68} \mathrm{Ga}$-DOTATATE MIP images of a patient before (a) and 12 months after (b) SRT with ${ }^{177}$ Lu-Dotatate. With excellent response of most of the bone metastasis to therapy, without significant changes on CT
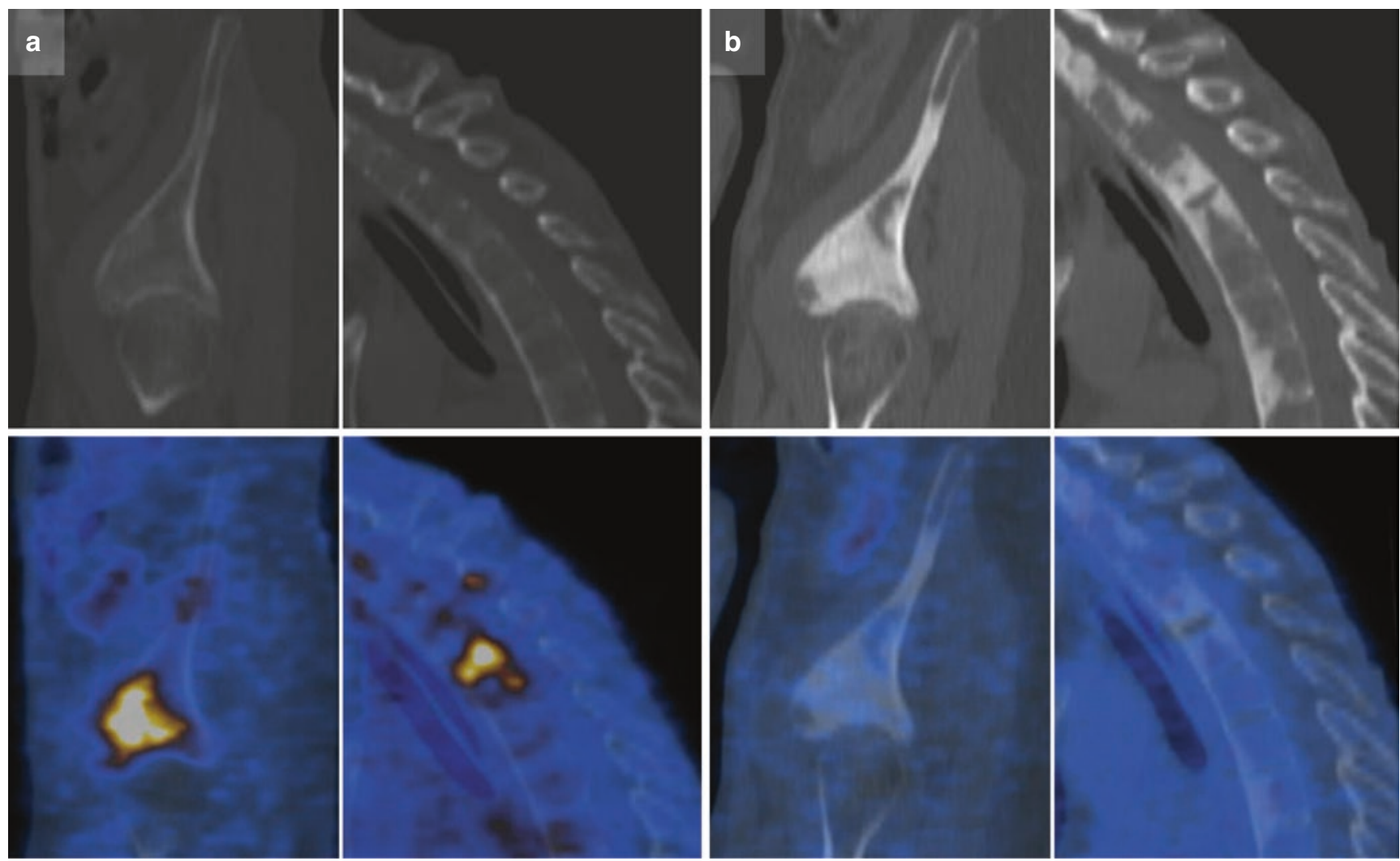

Fig. 4.7 Sagittal CT and PET/CTs of bone metastasis before (a) and after (b) chemotherapy showing increasing and new sclerotic lesions with a complete decrease on FDG PET consistent with good metabolic response 


\subsubsection{Monitoring Metastasized Prostate Cancer with MRI and PET/CT}

The use of FDG PET/CT is very limited in prostate cancer patients since only a small subgroup of highly dedifferentiated tumors will have increased glucose uptake. With improvements in technology, the use of diffusion-weighted imaging (DWI) became feasible not only for small areas but for whole-body exams. First preliminary results showed that ADC values could not differentiate between responders and nonresponders in patients undergoing chemotherapy [59]. However, this technology is still in its infancy, and other groups showed a good correlation between decrease in PSA and increase in ADC values for patients under antiandrogen therapy [60]. Assessment of nodal, visceral, and osseous metastasis with one exam is possible with ${ }^{18} \mathrm{~F}$ - or ${ }^{11} \mathrm{C}$-choline or ${ }^{68} \mathrm{Ga}$-prostate-specific membrane antigen (PSMA) PET/CT. A good correlation between apoptosis and decrease in SUV on ${ }^{11} \mathrm{C}$-choline PET/CT scans was shown after neoadjuvant docetaxel chemotherapy and complete androgen blockade in locally advanced prostate cancer patients [61]. The prospective use of choline PET/CT for response assessment of standardized docetaxel first-line chemotherapy on the other hand showed no correlation between changes in choline uptake and clinical assessments of progression based on RECIST 1.1 and PSA values [62].
There is a fast-increasing use of ${ }^{68} \mathrm{Ga}$-PSMA PET/CT for early biochemical recurrence detection in prostate cancer patients. The clinical utility of ${ }^{68} \mathrm{Ga}$-PSMA PET for treatment response is not established (Fig. 4.8). First investigations showed promising results using ${ }^{68} \mathrm{Ga}$-PSMA PET to evaluate ${ }^{223} \mathrm{Ra}$ therapy response [63]. The use of ${ }^{68} \mathrm{Ga}$-PSMA PET for response assessment to androgen deprivation therapy (ADT) will need careful prospective evaluation, since preliminary in vitro results showed that ADT is increasing the cellular expression of PSMA; therefore, a novel "tumor flare" might be observed in these patients [64].

\section{Take-Home Messages}

- After neoadjuvant therapy, rectal cancer resectability is essential for treatment planning. Restaging MRI shows tumor regression and clearance of the mesorectal fascia. If nonoperative management is considered in very good responders, diffusionweighted MRI combined with digital rectal examination and endoscopy accurately assesses clinical complete response.

- Systemic therapy response assessment with imaging is generally based on morphology. However,
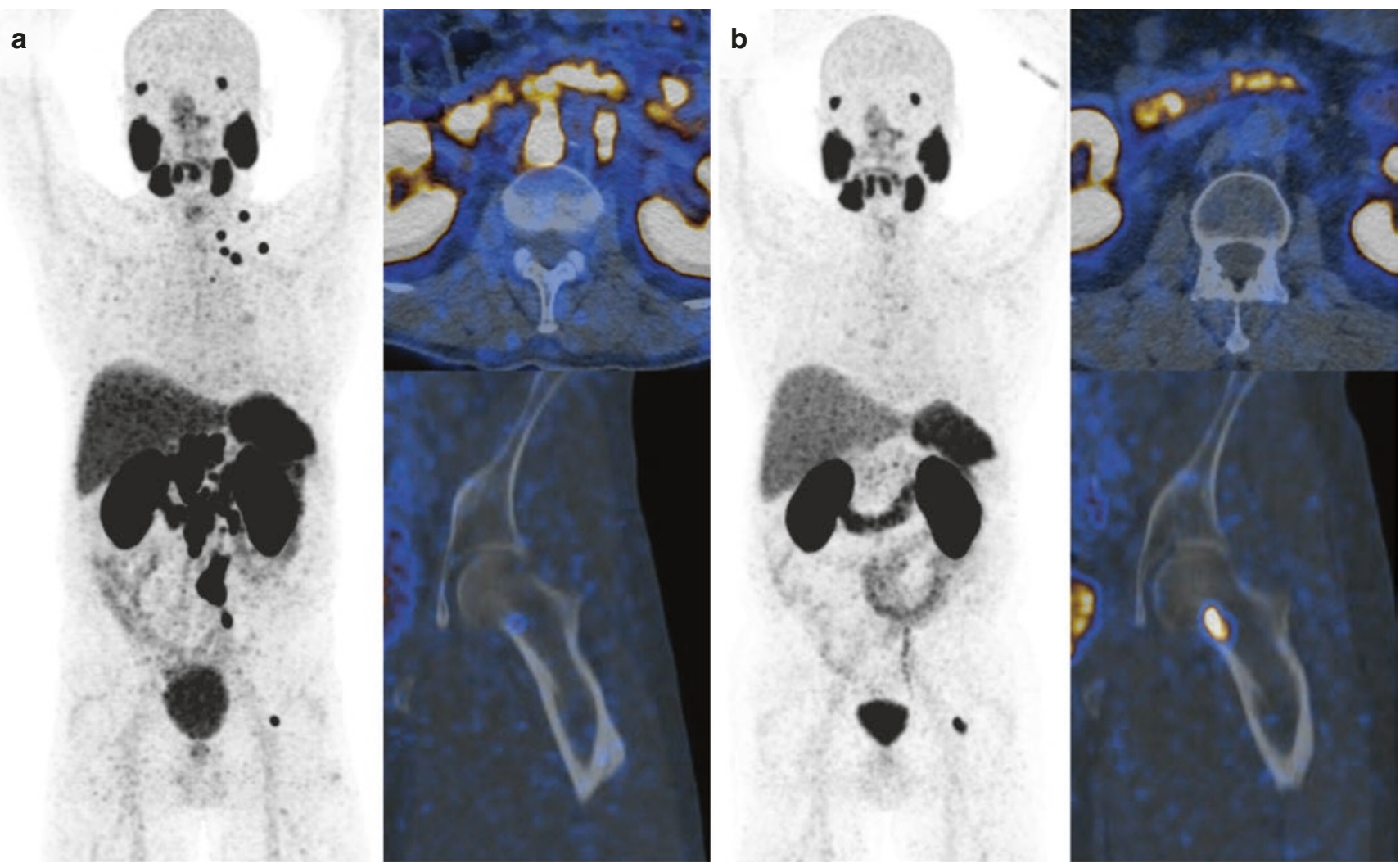

Fig.4.8 ${ }^{68} \mathrm{Ga}$-PSMA PET before (a) and after (b) two cycles of ${ }^{177}$ Lu-PSMA therapy with heterogeneous response: significant reduction in PSMA expression and size in all lymph node metastasis; however increase in PSMA expression and size of the bone metastasis 
with the introduction of noncytotoxic therapies, response patterns became very heterogeneous. Especially immunotherapy or local therapy of liver metastasis such as SIRT can lead to an initial pseudoprogression as a part of the response pattern with delayed morphologic response.

- PET can be used to identify response before morphologic changes are present in selected cases (GIST).

- Morphologic assessment of bone metastasis is only possible for lytic lesions with a soft tissue component. New sclerotic lesions on CT are not necessarily a sign of tumor progression but might be a sign of response of previously occult lesions.

\section{References}

1. Nagtegaal ID, Quirke P. What is the role for the circumferential margin in the modern treatment of rectal cancer? J Clin Oncol. 2008;26:303-12.

2. Beets-Tan RG, Beets GL, Vliegen RF, et al. Accuracy of magnetic resonance imaging in prediction of tumour-free resection margin in rectal cancer surgery. Lancet. 2001;357:497-504.

3. Brown G, Radcliffe AG, Newcombe RG, Dallimore NS, Bourne MW, Williams GT. Preoperative assessment of prognostic factors in rectal cancer using high-resolution magnetic resonance imaging. Br J Surg. 2003;90:355-64.

4. Extramural depth of tumor invasion at thin-section MR in patients with rectal cancer: results of the MERCURY study. Radiology. 2007;243:132-9.

5. Beets-Tan RGH, Lambregts DMJ, Maas M, et al. Magnetic resonance imaging for clinical management of rectal cancer: updated recommendations from the 2016 European Society of Gastrointestinal and Abdominal Radiology (ESGAR) consensus meeting. Eur Radiol. 2017. https://doi.org/10.1007/s00330-017-5026-2.

6. Vliegen RF, Beets GL, Lammering G, et al. Mesorectal fascia invasion after neoadjuvant chemotherapy and radiation therapy for locally advanced rectal cancer: accuracy of MR imaging for prediction. Radiology. 2008;246:454-62.

7. Kulkarni T, Gollins S, Maw A, Hobson P, Byrne R, Widdowson D. Magnetic resonance imaging in rectal cancer downstaged using neoadjuvant chemoradiation: accuracy of prediction of tumour stage and circumferential resection margin status. Color Dis. 2008; 10:479-89.

8. Habr-Gama A, Perez RO, Nadalin W, et al. Operative versus nonoperative treatment for stage 0 distal rectal cancer following chemoradiation therapy: long-term results. Ann Surg. 2004;240:711-7. discussion 717-718

9. Maas M, Beets-Tan RG, Lambregts DM, et al. Wait-and-see policy for clinical complete responders after chemoradiation for rectal cancer. J Clin Oncol. 2011;29:4633-40.

10. Beets-Tan RG, Beets GL. Rectal cancer: review with emphasis on MR imaging. Radiology. 2004;232:335-46.

11. van der Paardt MP, Zagers MB, Beets-Tan RG, Stoker J, Bipat S. Patients who undergo preoperative chemoradiotherapy for locally advanced rectal cancer restaged by using diagnostic MR imaging: a systematic review and meta-analysis. Radiology. 2013;269:101-12.

12. Dresen RC, Beets GL, Rutten HJ, et al. Locally advanced rectal cancer: MR imaging for restaging after neoadjuvant radiation therapy with concomitant chemotherapy. Part I. Are we able to predict tumor confined to the rectal wall? Radiology. 2009;252:71-80.

13. Barbaro B, Fiorucci C, Tebala C, et al. Locally advanced rectal cancer: MR imaging in prediction of response after preoperative chemotherapy and radiation therapy. Radiology. 2009;250:730-9.

14. Curvo-Semedo L, Lambregts DM, Maas M, et al. Rectal cancer: assessment of complete response to preoperative combined radiation therapy with chemotherapy--conventional MR volumetry versus diffusion-weighted MR imaging. Radiology. 2011;260:734-43.

15. Lambregts DM, Vandecaveye V, Barbaro B, et al. Diffusionweighted MRI for selection of complete responders after chemoradiation for locally advanced rectal cancer: a multicenter study. Ann Surg Oncol. 2011;18(8):2224-31.

16. Napoleon B, Pujol B, Berger F, Valette PJ, Gerard JP, Souquet JC. Accuracy of endosonography in the staging of rectal cancer treated by radiotherapy. Br J Surg. 1991;78:785-8.

17. Mezzi G, Arcidiacono PG, Carrara S, et al. Endoscopic ultrasound and magnetic resonance imaging for re-staging rectal cancer after radiotherapy. World J Gastroenterol. 2009;15:5563-7.

18. Radovanovic Z, Breberina M, Petrovic T, Golubovic A, Radovanovic D. Accuracy of endorectal ultrasonography in staging locally advanced rectal cancer after preoperative chemoradiation. Surg Endosc. 2008;22:2412-5.

19. Pastor C, Subtil JC, Sola J, et al. Accuracy of endoscopic ultrasound to assess tumor response after neoadjuvant treatment in rectal cancer: can we trust the findings? Dis Colon Rectum. 2011;54:1141-6.

20. Bipat S, Glas AS, Slors FJ, Zwinderman AH, Bossuyt PM, Stoker J. Rectal cancer: local staging and assessment of lymph node involvement with endoluminal US, CT, and MR imaging - a metaanalysis. Radiology. 2004;232:773-83.

21. Organization WH. WHO handbook for reporting results of cancer treatment; 1979.

22. Eisenhauer EA, Therasse P, Bogaerts J, et al. New response evaluation criteria in solid tumours: revised RECIST guideline (version 1.1). Eur J Cancer. 2009;45:228-47.

23. Seymour L, Bogaerts J, Perrone A, et al. iRECIST: guidelines for response criteria for use in trials testing immunotherapeutics. Lancet Oncol. 2017;18:e143-52.

24. Chiou VL, Burotto M. Pseudoprogression and immune-related response in solid tumors. J Clin Oncol. 2015;33:3541-3.

25. Wong ANM, McArthur GA, Hofman MS, Hicks RJ. The advantages and challenges of using FDG PET/CT for response assessment in melanoma in the era of targeted agents and immunotherapy. Eur J Nucl Med Mol Imaging. 2017;44:67-77.

26. Rymer B, Curtis NJ, Siddiqui MR, Chand M. FDG PET/CT can assess the response of locally advanced rectal cancer to neoadjuvant chemoradiotherapy: evidence from meta-analysis and systematic review. Clin Nucl Med. 2016;41:371-5.

27. Wahl RL, Jacene H, Kasamon Y, Lodge MA. From RECIST to PERCIST: evolving considerations for PET response criteria in solid tumors. J Nucl Med. 2009;50(Suppl 1):122S-50S.

28. Pinker K, Riedl C, Weber WA. Evaluating tumor response with FDG PET: updates on PERCIST, comparison with EORTC criteria and clues to future developments. Eur J Nucl Med Mol Imaging. 2017;44:55-66.

29. van Oosterom AT, Judson I, Verweij J, et al. Safety and efficacy of imatinib (STI571) in metastatic gastrointestinal stromal tumours: a phase I study. Lancet. 2001;358:1421-3.

30. Joensuu $\mathrm{H}$. Treatment of inoperable gastrointestinal stromal tumor (GIST) with Imatinib (Glivec, Gleevec). Med Klin (Munich). 2002;97(Suppl 1):28-30.

31. Van den Abbeele AD. The lessons of GIST--PET and PET/CT: a new paradigm for imaging. Oncologist. 2008;13(Suppl 2):8-13.

32. Shinagare AB, Jagannathan JP, Krajewski KM, Ramaiya NH. Liver metastases in the era of molecular targeted therapy: new faces of treatment response. AJR Am J Roentgenol. 2013;201:W15-28.

33. Choi H, Charnsangavej C, Faria SC, et al. Correlation of computed tomography and positron emission tomography in patients with metastatic gastrointestinal stromal tumor treated at a single institution with imatinib mesylate: proposal of new computed tomography response criteria. J Clin Oncol. 2007;25:1753-9. 
34. Apfaltrer P, Meyer M, Meier C, et al. Contrast-enhanced dualenergy CT of gastrointestinal stromal tumors: is iodine-related attenuation a potential indicator of tumor response? Investig Radiol. 2012;47:65-70.

35. Tang L, Zhang XP, Sun YS, et al. Gastrointestinal stromal tumors treated with imatinib mesylate: apparent diffusion coefficient in the evaluation of therapy response in patients. Radiology. 2011;258:729-38.

36. Casali PG, Blay JY, Bertuzzi A, et al. Gastrointestinal stromal tumours: ESMO clinical practice guidelines for diagnosis, treatment and follow-up. Ann Oncol. 2014;25:21-6.

37. Holdsworth CH, Badawi RD, Manola JB, et al. CT and PET: early prognostic indicators of response to imatinib mesylate in patients with gastrointestinal stromal tumor. AJR Am J Roentgenol. 2007;189:W324-30.

38. Benjamin RS, Schoffski P, Hartmann JT, et al. Efficacy and safety of motesanib, an oral inhibitor of VEGF, PDGF, and kit receptors, in patients with imatinib-resistant gastrointestinal stromal tumors. Cancer Chemother Pharmacol. 2011;68:69-77.

39. Farag S, de Geus-Oei LF, Van der Graaf WT, et al. Early response evaluation by 18 F-FDG-PET influences management in gastrointestinal stromal tumor (GIST) patients treated with imatinib with neo-adjuvant intent. J Nucl Med. 2017. https://doi.org/10.2967/ jnumed.117.196642.

40. Stubbs RS, Cannan RJ, Mitchell AW. Selective internal radiation therapy with 90yttrium microspheres for extensive colorectal liver metastases. J Gastrointest Surg. 2001;5:294-302.

41. Boppudi S, Wickremesekera SK, Nowitz M, Stubbs R. Evaluation of the role of CT in the assessment of response to selective internal radiation therapy in patients with colorectal liver metastases. Australas Radiol. 2006;50:570-7.

42. Morsbach F, Pfammatter T, Reiner CS, et al. Computed tomographic perfusion imaging for the prediction of response and survival to transarterial radioembolization of liver metastases. Investig Radiol. 2013;48:787-94.

43. Reiner CS, Morsbach F, Sah BR, et al. Early treatment response evaluation after yttrium-90 radioembolization of liver malignancy with CT perfusion. J Vasc Interv Radiol. 2014;25:747-59.

44. Lee SH, Lee JM, Kim KW, et al. Dual-energy computed tomography to assess tumor response to hepatic radiofrequency ablation: potential diagnostic value of virtual noncontrast images and iodine maps. Investig Radiol. 2011;46:77-84.

45. Schmeel FC, Simon B, Sabet A, et al. Diffusion-weighted magnetic resonance imaging predicts survival in patients with liverpredominant metastatic colorectal cancer shortly after selective internal radiation therapy. Eur Radiol. 2017;27:966-75.

46. Wong CY, Qing F, Savin M, et al. Reduction of metastatic load to liver after intraarterial hepatic yttrium-90 radioembolization as evaluated by $\left[{ }^{18} \mathrm{~F}\right]$ fluorodeoxyglucose positron emission tomographic imaging. J Vasc Interv Radiol. 2005;16:1101-6.

47. Szyszko T, Al-Nahhas A, Canelo R, et al. Assessment of response to treatment of unresectable liver tumours with $90 \mathrm{Y}$ microspheres: value of FDG PET versus computed tomography. Nucl Med Commun. 2007;28:15-20.

48. Annunziata S, Treglia G, Caldarella C, Galiandro F. The role of 18F-FDG-PET and PET/CT in patients with colorectal liver metastases undergoing selective internal radiation therapy with yttrium-90: a first evidence-based review. ScientificWorldJournal. 2014;2014:879469.
49. Fendler WP, Philippe Tiega DB, Ilhan H, et al. Validation of several SUV-based parameters derived from 18F-FDG PET for prediction of survival after SIRT of hepatic metastases from colorectal cancer. J Nucl Med. 2013;54:1202-8.

50. Dierckx R, Maes A, Peeters M, Van De Wiele C. FDG PET for monitoring response to local and locoregional therapy in HCC and liver metastases. Q J Nucl Med Mol Imaging. 2009;53:336-42.

51. Pavel ME, Hainsworth JD, Baudin E, et al. Everolimus plus octreotide long-acting repeatable for the treatment of advanced neuroendocrine tumours associated with carcinoid syndrome (RADIANT-2): a randomised, placebo-controlled, phase 3 study. Lancet. 2011;378:2005-12.

52. Strosberg J, El-Haddad G, Wolin E, et al. Phase 3 trial of 177Lu-Dotatate for Midgut neuroendocrine tumors. N Engl J Med. 2017;376:125-35.

53. Hicks RJ, Kwekkeboom DJ, Krenning E, et al. ENETS Consensus Guidelines for the Standards of Care in Neuroendocrine Neoplasia: peptide receptor radionuclide therapy with radiolabeled somatostatin analogues. Neuroendocrinology. 2017;105(3):295-309.

54. Haug AR, Auernhammer CJ, Wangler B, et al. 68Ga-DOTATATE $\mathrm{PET} / \mathrm{CT}$ for the early prediction of response to somatostatin receptormediated radionuclide therapy in patients with well-differentiated neuroendocrine tumors. J Nucl Med. 2010;51:1349-56.

55. de Bono JS, Logothetis CJ, Molina A, et al. Abiraterone and increased survival in metastatic prostate cancer. N Engl J Med. 2011:364:1995-2005.

56. Parker C, Nilsson S, Heinrich D, et al. Alpha emitter radium-223 and survival in metastatic prostate cancer. NEngl J Med. 2013;369:213-23.

57. Costelloe CM, Chuang HH, Madewell JE, Ueno NT. Cancer response criteria and bone metastases: RECIST 1.1, MDA and PERCIST. J Cancer. 2010;1:80-92.

58. Hamaoka T, Madewell JE, Podoloff DA, Hortobagyi GN, Ueno NT. Bone imaging in metastatic breast cancer. J Clin Oncol. 2004;22:2942-53.

59. Messiou C, Collins DJ, Giles S, de Bono JS, Bianchini D, de Souza NM. Assessing response in bone metastases in prostate cancer with diffusion weighted MRI. Eur Radiol. 2011;21:2169-77.

60. Reischauer C, Froehlich JM, Koh DM, et al. Bone metastases from prostate cancer: assessing treatment response by using diffusionweighted imaging and functional diffusion maps--initial observations. Radiology. 2010;257:523-31.

61. Schwarzenbock SM, Knieling A, Souvatzoglou M, et al. $\left[{ }^{11} \mathrm{C}\right]$ choline PET/CT in therapy response assessment of a neoadjuvant therapy in locally advanced and high risk prostate cancer before radical prostatectomy. Oncotarget. 2016;7:63747-57.

62. Schwarzenbock SM, Eiber M, Kundt G, et al. Prospective evaluation of $\left[{ }^{11} \mathrm{C}\right]$ choline PET/CT in therapy response assessment of standardized docetaxel first-line chemotherapy in patients with advanced castration refractory prostate cancer. Eur J Nucl Med Mol Imaging. 2016;43:2105-13.

63. Bieth M, Kronke M, Tauber R, et al. Exploring new multimodal quantitative imaging indices for the assessment of osseous tumour burden in prostate cancer using 68Ga-PSMA-PET/CT. J Nucl Med. 2017;58(10):1632-7.

64. Meller B, Bremmer F, Sahlmann CO, et al. Alterations in androgen deprivation enhanced prostate-specific membrane antigen (PSMA) expression in prostate cancer cells as a target for diagnostics and therapy. EJNMMI Res. 2015;5:66.

Open Access This chapter is licensed under the terms of the Creative Commons Attribution 4.0 International License (http://creativecommons. org/licenses/by/4.0/), which permits use, sharing, adaptation, distribution and reproduction in any medium or format, as long as you give appropriate credit to the original author(s) and the source, provide a link to the Creative Commons license and indicate if changes were made.

The images or other third party material in this book are included in the book's Creative Commons license, unless indicated otherwise in a credit line to the material. If material is not included in the book's Creative Commons license and your intended use is not permitted by statutory regulation or exceeds the permitted use, you will need to obtain permission directly from the copyright holder. 